\title{
Non-Booking For Antenatal Care And Risks For Vertical HIV Transmission Among Women In Chitungwiza, Zimbabwe: A Cross-Sectional Study.
}

\section{Patricia Mandima ( $\nabla$ pmandima@uz-ctrc.org)}

University of Zimbabwe- Clinical Trials Research Centre

Nikki Schaay

University of the Western School of Public Health

\section{Bernard Ngara}

University of Zimbabwe

\section{Martina Lembani}

University of the Western School of Public Health

\section{Research Article}

Keywords: Booking, Pregnancy, HIV, PMTCT, Women

Posted Date: January 21st, 2022

DOI: https://doi.org/10.21203/rs.3.rs-1224535/v1

License: @ (i) This work is licensed under a Creative Commons Attribution 4.0 International License. Read Full License 


\section{Abstract}

Background: The success of prevention of mother to child transmission of HIV (PMTCT) dependents on pregnant women accessing antenatal care (ANC) services. Failure to book for ANC through the course of pregnancy presents a missed opportunity for PMTCT and a high risk for maternal HIV transmission. Whilst unbooking was about $6 \%$ in Zimbabwe, it is important to determine the local burden of women both un-booked for ANC and living with HIV among pregnant women. in Chitungwiza city This study aimed at determining the proportion of women un-booked for antenatal care and among them, the proportion of women who were with HIV and to identify risk factors associated with un-booking in Chitungwiza city in Zimbabwe,

Methods: A cross-sectional study was conducted involving a review of clinic records for 4400 women who received postnatal care at all 4 maternity clinics in Chitungwiza city between 01 January 2017 and 31 December 2017. The Chi-square test was used to determine association between participants' booking status and socio-demographic variables. Multiple logistic regression was used to determine risk factors associated with booking status while adjusting for other study variables. All statistics tests' decisions were concluded at $5 \%$ level of significance. All data analysis was performed using STATA (version 13) statistical package.

Results: A total of $19 \%$ of pregnant women were un-booked for ANC, while a total of $3 \%$ of the women were both un-booked and living with HIV. The women with HIV were 0.24 times less likely to book for ANC than HIV negative women, adjusted OR $0.76,95 \% \mathrm{Cl}(0.61-0.98) \mathrm{P}=0.037$. Women aged $20-34$ years were 1.3 times more likely to book than the teenagers, adjusted $\mathrm{OR}$ $1.3,95 \% \mathrm{CL}(1.04-1.62), \mathrm{P}=0.022$.

Conclusion: Not booking for ANC is a problem in the city of Chitungwiza, affecting access to PMTCT services. The results from this study illustrate the need to use local data for local planning rather than national data.

\section{Background}

At the end of 2020, approximately 38 million people were living with human immunodeficiency virus (HIV) and of these, 1 million were children aged 0 to 9 years $^{1}$. In the same year, 1.5 million people became newly infected with HIV around the world this included 160000 children under the age of nine ${ }^{1}$

Approximately 1.3 million women living with HIV got pregnant in $2018^{2}$ and Mother- to- Child Transmission of HIV (MTCT) inutero or through breastfeeding is responsible for over $90 \%$ of all paediatric HIV infections. ${ }^{3}$ Prevention of Mother-to-Child Transmission of HIV (PMTCT) is therefore critical in the prevention of paediatric HIV infection. In an attempt to prevent HIV infection occurring in children, the World Health organisation (WHO) recommends a range of strategies, namely: the prevention of HIV in women of childbearing age, the prevention of unwanted pregnancies, PMTCT with anti-retroviral therapy (ART) in HIV positive pregnant women and infant prophylaxis for HIV exposed infants ${ }^{4}$. Antenatal care (ANC) plays a vital role in one of these strategies, namely, PMTCT, since it is the entry point for pregnant women into health care services. Women, who for various reasons fail to utilise ANC, would unfortunately have missed the opportunity to fully access PMTCT and are at high risk for maternal HIV transmission.

This study aimed at determining the proportion of women un-booked for antenatal care and among them, the proportion of women who were HIV positive and to identify risk factors associated with un-booking in the urban city of Chitungwiza in Zimbabwe,

Antenatal care refers to the ongoing health services provided to women during pregnancy in the formal health system. It is meant to screen out pregnant women for potential obstetric complications, non-communicable and infectious diseases and provide therapeutic and preventive interventions in order to minimise maternal and fatal adverse pregnancy outcomes ${ }^{5}$. In 2002, the WHO issued a set of ANC guidelines for developing countries which incorporated what was proposed in relation to the prevention of mother to child transmission of HIV. ${ }^{5}$

Page 2/13 
Henceforth, ANC became the focal point for access to PMTCT by pregnant women. ${ }^{6}$ Unfortunately, about $14 \%$ of pregnant women worldwide have no access to a single ANC visit with trained healthcare workers. ${ }^{7}$ In Zimbabwe, the HIV prevalence among pregnant women is $16.7 \%$ and about $7 \%$ of all pregnant women in Zimbabwe fail to access ANC services throughout pregnancy for various reasons. ${ }^{8}$ These women, un-booked for ANC, unfortunately miss the opportunity to fully utilise PMTCT services and those with HIV become very high risk for MTCT. Without PMTCT the risk for MTCT is 15 to $45 \%{ }^{9}$ but can be as low as below $1 \%$ with PMTCT. ${ }^{10}$

Many factors have been described which affect access to antenatal care. They include women's perceptions on the importance of ANC and health problems during pregnancy. It has been suggested that marital status, parity, health worker - related factors, the costs of ANC and the fear of HIV testing were some of the factors influential in the utilisation of antenatal care services. ${ }^{11}$

A systematic review by Simkhada (2008) further identified maternal education, husband's education, availability of ANC services, household income, women's employment status, media exposure and having a history of obstetric complications along with a women's marital status, as factors affecting access to ANC. Cultural beliefs and ideas about pregnancy also influenced ANC utilization. ${ }^{12}$ Many studies have shown that poor socio-economic status is associated with poor use of antenatal services. ${ }^{13,14,15}$ For example, attending ANC is associated with costs even in facilities where the service is offered free. Such costs include those required for transport and the time spent at ANC.

Women who have completed secondary school or those with a tertiary education are noted, in many studies, as better utilisers of antenatal care services compared to those with no education and those who did not complete primary or secondary education. ${ }^{16,17,18}$ Educated women may have a better perception of the importance of ANC, have a better understanding of health education messages and are less intimidated to approach health care workers. ${ }^{11}$ Similarly, it has been noted that professional women are good users of antenatal care services compared to unemployed women. ${ }^{12,17}$ Women who get pregnant at ages less than 20 are usually not financially independent, unmarried and have not yet completed secondary or tertiary education and have been identified as poor utilisers of antenatal care services. In some settings, this can be worsened by poor pregnancy disclosure and social stigma. ${ }^{11,16}$

Researchers have noted that lower parity, a planned pregnancy, and past pregnancy complications were associated with better utilisation of $\mathrm{ANC} \cdot 16,17$

Interestingly, most studies focusing on the risk factors associated with poor utilisation of ANC tend to focus on the comparison between early bookers and late bookers and do not focus on the risk factors for not booking at all. Galvin (2001) showed that non-bookers were likely to be of lower socio-economic status compared to booked women in Harare, Zimbabwe. However, this study was done at a referral hospital where most of the non-bookers had been referred from rural areas which does not represent the urban Chitungwiza population 18

Despite the fact that anti-retroviral drugs have been made available free of charge in the public health sector since 2002 and that HIV care is decentralized so that PMTCT services are available at all maternity health centres in Zimbabwe, some pregnant women appear to still find it difficult to access these services. ${ }^{8}$ These women either present at maternity clinics as unbooked clients during labour or well after giving birth without having had a single contact with antenatal care services prior to the labour or delivery. Those newly diagnosed with HIV would then have missed taking ART during pregnancy and one of the obvious results of this is the children born are at high risk (15-45\%) of contracting HIV, ${ }^{9}$ when such could have been prevented.

Whilst there is the Harare provincial data showing an un-booked proportion of $6 \%,{ }^{8}$ the local burden of un-booked women in the city of Chitungwiza is not known. The proportion of these un-booked women who also have HIV and have missed utilisation of all PMTCT services during pregnancy, is also unknown. A study by Chadwick et al, which recruited unbooked 
postpartum women with HIV indicated that the proportion of unbooked women in Chitungwiza could be way higher than the $6 \%$ estimated for Harare province where Chitungwiza is located. ${ }^{19}$

This study therefore aimed at determining the proportion of women un-booked for antenatal care until delivery and among them, the proportion of women who were HIV positive, and to identify some of the risk factors for un-booking in the city of Chitungwiza. Determining the local burden of non-utilisation of ANC services is essential in determining what local health promotion strategies and health service actions and resources are needed to bring such pregnant women into care.

\section{Methods}

\section{Study design}

The study used an analytical cross-sectional design based on retrospective review of clinic records of women who delivered and/or received postnatal care at the 4 maternity clinics in Chitungwiza between 01 January 2017 and 31 December 2017. This design was chosen in order to determine at one point frequencies and distribution of exposures and outcome variables over a one year period in a population of postpartum women and to further investigate the relationships between exposure and outcome variables ${ }^{20}$. The study was to determine frequency of unbooking and outcome variables among the postpartum women and to assess the association between booking status and HIV status, age and clinic attended.

\section{Description of study setting}

The study was carried out in Chitungwiza which is the third most populous city in Zimbabwe, situated about $30 \mathrm{~km}$ south of the capital city, Harare. Chitungwiza is an urban city surrounded by urban Harare on the northern side, peri-urban informal settlements on the western side, then a rural setting on the south and eastern sides. It has a population of 356,840 and an annual growth rate of $1 \%$. About $64 \%$ of the population is comprised of adolescents and adults over 15 years of age, among whom $30 \%$ are women of child-bearing age (15-49 years) (Unpublished Chitungwiza city health statistics). The overall adult prevalence of HIV in Chitungwiza in the 15-49-year age group is estimated to be 15\%, with women harbouring higher burden of disease at $18 \%$ prevalence in comparison with $12 \%$ among men. The prevalence among antenatal clinic attendees is $16 \%$, with Chitungwiza having high teenage pregnancy rate with $27 \%$ of teenagers having begun childbearing (unpublished data). The city is divided into 4 urban sub-districts, Seke North, Seke South, Zengeza and St Mary's and each sub-district is serviced by a polyclinic with a similar name as the sub-district. The maternity side of the clinics is staffed by midwives who refer complicated cases to the government-run Chitungwiza General Hospital situated about 4-6 km from each clinic. The clinics have been offering PMTCT services since 2002 to the Chitungwiza urban and peri-urban community. There is also a private hospital in the city and many private doctors. The study was carried out in 2018 at the 4 polyclinics through collection of data from clinical records of all the women who delivered at the clinics from 01 January 2017 to 31 December 2017.

\section{Study population and sampling}

The study population included all women who were registered to have received postnatal care at the 4 study maternity clinics between 01 January and 31 December 2017.

\section{Sample size determination}

The Dobson's sample size calculation was used to determine minimum sample size, using level of significance of $5 \%$ and the expected proportion (p) of booked women in the target population of $93 \%$ and margin of error of $5 \%$. The minimum number of participants required from each clinic at $5 \%$ level of significance, assuming $p=93 \%{ }^{8}$ and $5 \%$ error of margin was 100 . The total minimum sample size required was 400 participants for all clinics. However, since the clinic records were already available, a total population sample size of 4400 was used in the analysis. Use of the largest available sample was done in order to improve the validity and reliability of the results by further reducing the impact of chance

\section{Inclusion criteria}


Women who had delivered at term at any one of the 4 study maternity clinics between 01 January and 31 December of 2017 were included.

Women presenting at any one of the 4 study maternity clinics after term home deliveries between 01 January and 31 December of 2017 were also included.

Women whose term infants were delivered as still births or who had died soon after birth were included.

\section{Exclusion criteria}

Women with miscarriages, abortions and preterm deliveries.

Women who were referred, during labour, for further care and did not deliver at the clinics

\section{Data collection}

Data collection started after receiving approval from UWC IRB, MRCZ and from Chitungwiza City Ethics Committee. Upon receiving permission to perform the study at the clinics by the Chitungwiza City Health Department, the clinic managers of the 4 clinics were notified about the study and requested to give study staff access to maternity records. A nurse visited each clinic once weekly from August to November 2018 to access the maternity records and to abstract data from the maternity registers to a data extraction sheet. Data on Age, HIV status, and clinic attended were collected. Names were not recorded during data abstract; numbering was used instead.

\section{Data Management}

Data collected on the data extraction tool was checked for completeness after which it was entered onto an excel spreadsheet by a data entry cleck. Data cleaning was repeated after entry onto the Excel spreadsheet to ensure completeness and accuracy of the data. Data entry and quality check continued from February 2019 to April 2019

\section{Data analysis}

All the data was categorized and firstly summarized using frequencies and percentages. Chi-square test was used in bivariate analysis to determine association between participants' booking status and age, booking status and HIV status, and booking status and attended clinic. Associations showing $P$ values of less than 0.05 were further analysed by multiple logistic regression to determine the association with booking status while adjusting for other study variables. All statistic tests decisions were concluded at $5 \%$ level of significance. All statistical analyses were performed in STATA software package version 13.

\section{Results}

\section{Description of study data}

The results in this section show the univariate analysis of the descriptive characteristics of the 4400 women who delivered and/or received postnatal care at the 4 maternity clinics between January and December 2017.

A total of 4400 women delivered and/or received postnatal care at the 4 maternity clinics in 2017 that were included in the study. Seke South clinic attended to the largest number of women. Of the 4400 women, 1397 (32\%) were from Seke South clinic, 1264 (28\%) were from Seke North clinic, 1012 (23\%) from St Mary clinic and 727 (17\%) were from Zengeza clinic. The most common age group was the $20-34$ years group, contributing $75 \%$ of the population with 3304 women, $14 \%$ (597 women) were less than 20 years of age and 11\% (499 women) were above 34 years of age. Of the 4400 women, 820 (19\%) were unbooked for ANC. Out of the 4400 women, a total of 470 were HIV positive, giving an ANC HIV prevalence of $11 \%$. See Table 1 below. 
Table 1

General description of the women who delivered and/or received postnatal care in Chitungwiza clinics.

\begin{tabular}{|ll|}
\hline Variable & Frequency (percentage in study population) \\
\hline Clinic attended & \\
\hline Seke North & $1264(28)$ \\
Seke South & $1397(32)$ \\
\hline St Marry & $1012(23)$ \\
\hline Zengeza & $727(17)$ \\
\hline Age in years & \\
\hline$<20$ & $597(14)$ \\
\hline $20-34$ & $3304(75)$ \\
\hline$>34$ & $499(11)$ \\
\hline HIV status & \\
\hline Negative & $3930(89)$ \\
\hline Positive & $470(11)$ \\
\hline Booking status & \\
\hline Booked & $3580(81)$ \\
\hline Un-booked & $820(19)$ \\
\hline
\end{tabular}

Factors associated with booking status without adjustment of the effect of other study variables.

Table 2 below shows results of bivariate analysis with chi-squared test to show factors associated with unbooking among the 4400 postnatal women at the 4 maternity clinics. Only those factors with $p$ values $<0.05$ have an association with booking.

\section{Clinic attended}

The percentage of unbooking was highest at St Mary's clinic, compared to other clinics. A total of 236 out of 776 (23\%) of those attended at St Mary's clinic were un-booked, 218 of the 1046 (17\%) from Seke North were un-booked, 253 of the 1144 (17\%) from Seke South and 113 of 614 (16\%) from Zengeza clinic were un-booked. There was more unbooking at St Mary's clinic compared to other clinics and the difference was statistically significant $(P<0.001)$ before adjusting for other study variables.

\section{Age}

Unbooking was highest among the younger and the older participants. Among the 597 participants aged less than 20 years, $136(23 \%)$ were un-booked and 115 of the $499(23 \%)$ women aged over 34 years were un-booked. Only $17 \%$ (567 of the 3304$)$ women aged 20-34 years were un-booked. Unbooking was lowest in the 20-34 years age group and difference was significant $(P<0.001)$ before adjusting for other study variables.

\section{HIV status}

Of the 820 un-booked women, 118 (14\%), were HIV positive. HIV infection was higher among un-booked women who delivered outside the health system. Out of the 207 un-booked women who delivered at home, 47 of them (23\%) were HIV positive. Out of the overall sample of $4400,118(3 \%)$ women were both un-booked and HIV positive. Only $18 \%$, (702 out of the 3930) of HIV 
negative women were un-booked, compared to $25 \%$ who were un-booked among the HIV positive women (118 of 407$)$, showing an association between unbooking and HIV infection $(P<0.001)$ before adjusting for other study variables.

Table 2

Chi-square test analysis to determine factors associated with booking

\begin{tabular}{|llll|}
\hline Variable & \multicolumn{2}{l}{ Booking Status } & p-value \\
\cline { 2 - 3 } & Booked & Un-booked & \\
\hline Clinic attended, $n(\%)$ & & & \\
\hline Seke North & $1046(83)$ & $218(17)$ & $<0.001$ \\
\hline Seke South & $1144(83)$ & $253(17)$ & \\
\hline St Marry & $776(77)$ & $236(23)$ & \\
\hline Zengeza & $614(84)$ & $113(16)$ & \\
\hline Age in years, $\mathbf{n}(\%)$ & & & \\
\hline$<20$ & $461(77)$ & $136(23)$ & $<0.001$ \\
\hline $20-34$ & $2737(83)$ & $567(17)$ & \\
\hline$>34$ & $384(77)$ & $115(23)$ & \\
\hline HIV status, $n(\%)$ & & & \\
\hline Negative & $3228(82)$ & $702(18)$ & $<0.001$ \\
\hline Positive & $352(75)$ & $118(25)$ & \\
\hline
\end{tabular}

Multivariate analysis to determine factors associated with booking after adjustment of the effect of other study variables.

A multivariate analysis was performed on all the variables which showed associated with booking in the bivariate analysis as shown in Table 3. This is done to further describe the relationship with $\mathrm{OR}$ and $\mathrm{Cl}$ and to adjust for confounding.

The HIV positive women were 0.24 times less likely to book than HIV negative women, Adjusted OR $0.76,95 \% \mathrm{Cl}(0.61-0.98)$ $\mathrm{P}=0$. 037. Women attended at St Mary's clinic were $23 \%$ less likely to book compared to Seke North women, Adjusted OR 0.77 , $95 \% \mathrm{Cl}(0.62-0.96), \mathrm{P}=0.020$. Women aged $20-34$ years were 1.3 times more likely to book than those aged $<20$ years, Adjusted OR $1.3,95 \%$ CL (1.04-1.62), $P=0.022$ 
Table 3

Multivariate logistic regression to determine factors associated with booking

\begin{tabular}{|llll|}
\hline Variable & Adjusted OR & $95 \% \mathrm{Cl}$ & p-value \\
\hline HIV Status & & & \\
negative & Reference & & \\
\hline positive & 0.76 & $0.61-0.98$ & 0.037 \\
\hline Clinic attended & & & \\
\hline Seke North & Reference & & \\
\hline Seke South & 0.85 & $0.69-1.05$ & 0.138 \\
\hline St Mary's & 0.77 & $0.62-0.96$ & 0.020 \\
\hline Zengeza & 0.98 & $0.76-1.27$ & 0.902 \\
\hline Age & & & \\
\hline$<20$ years & Reference & & \\
\hline $20-34$ years & 1.3 & $1.04-1.62$ & 0.022 \\
\hline$>34$ & 1.12 & $0.83-1.51$ & 0.464 \\
\hline
\end{tabular}

\section{Discussion}

The study identified the burden of unbooking for ANC in Chitungwiza to be $19 \%$. This burden is much higher and even triples the $6 \%$ unbooking suggested for Harare province where Chitungwiza is located. ${ }^{8}$. Percentages of women not utilizing ANC of greater than $19 \%$ have been reported for some Sub Saharan countries. A study involving 235,207 women who have had at least one birth within five years preceding Demographic and Health surveys of 31 Sub-Saharan Africa conducted between 2010 and 2018 showed that $13 \%$ of the women did not book for ANC. Countries with the highest percentage of women who did not book for ANC during pregnancy include Nigeria (26.1\%) Ethiopia (34.8\%) and Chad (41.8\%). The lowest percentages of less than $1 \%$ were reported for Rwanda, Gambia and Burundi. Although Harare provincial data on the percentage of un-booked women $\mathrm{f}$ are available from the 2015 ZDHS, there was no available local level data for the burden of unbooking specifically for Chitungwiza. These data are important for local outreach and health service planning and the intention of this study was to contribute to providing such local-level data. It must be borne in mind that the ZDHS results are based on women responding to a question on their booking status on the last baby they had in the past 5 years. According to the ZDHS 2015, "women who had a live birth in the 5 years preceding the survey were asked a number of questions about maternal and child health care. For the last live birth in that period, mothers were asked about antenatal care (ANC) during the pregnancy, assistance during delivery, the location of the delivery, and the timing of postnatal care." 8 In this case, the desire to please the interviewer and recall bias cannot be ruled out and could result in an underestimate of the problem of unbooking based on experiences with un-booked women in the city. ${ }^{19}$ It was the intention of this study to provide more reliable local data on the burden of unbooking - based on a more reliable method i.e. using delivery records.

The available national data on ANC access is not linked to antenatal HIV prevalence data, hence national, provincial, and local data on the percentage of un-booked pregnant women with HIV infection is not readily available. In this study, $3 \%$ of all women delivering and/or receiving postnatal care at Chitungwiza maternity clinics were both un-booked and living with HIV. These are women who did not access PMTCT services offered during ANC and were at high risk for perinatal HIV transmission. Unless such women are identified and brought into care, children will continue to be born with HIV amidst tried and tested HIV 
prevention measures. Though there are data on HIV seroprevalence among unbooked women, to our knowledge, this is the first study to identify the percentage of pregnant women who were both unbooked and living with HIV in Zimbabwe.

The study also found that the ANC prevalence of HIV was $11 \%$ This percentage was lower than the Zimbabwe national 2015 ANC prevalence of $16 \%{ }^{8}$ The lower percentage could be explained by the declining national HIV incidence which went down from $1 \%$ in 2015 to $0.54 \%$ in $2017 .^{21}$

Unbooking was significantly higher in St Mary's compared to the other 3 clinics. This could be explained by the lower socioeconomic status of St Mary's as it services more peri-urban overcrowded informal settlements compared to other clinics. It is common knowledge that peri-urban informal settlements are associated with poverty and unemployment which negatively affect utilisation of health services requiring user fees. ${ }^{13,14,15,16,17,18}$

Women less than 20 years and those above 34 were less likely to book compared to the 20-34-year-olds and this was clinically significant. These results were in contrast with the 2015 ZDHS data which showed highest ANC attendance of $94.6 \%$ among those aged $<20$ years compared to $93 \%$ in those above 20 years. ${ }^{8}$

A major limitation of the study is that it was designed as a cross-sectional study and can therefore only capture association but not causation. The study was not able to capture all un-booked women from Chitungwiza as some women deliver at home under the care of traditional birth attendants and do not visit the antenatal clinics. As Chitungwiza is only $28 \mathrm{~km}$ from Harare (the capital of Zimbabwe), some women opt to seek ANC at Harare clinics and others in private centres in Chitungwiza, thus were not captured by the study and not represented in the study.

\section{Conclusion}

This study aimed to provide data on burden of unbooking specifically for Chitungwiza city and so inform the planning of local interventions that will prioritise efforts to improve ANC utilisation in the city. The burden of unbooking was found to be $19 \%$ which is much higher than the expected $6 \%$

The burden of unbooked women who were also living HIV, who could have also missed opportunity for PMTCT during pregnancy was also determined to be $3 \%$. Hence unbooking for ANC is still a contributing factor to the persistent perinatal HIV transmission.

Programs that target HIV positive women, pregnant teenagers and older pregnant women and women from peri-urban areas could help improve ANC attendance hence PMTCT uptake in Chitungwiza. Lessons can be learnt from Tanzania where the use of community health workers (CHWs) to identify pregnant women in the community and counselling on ANC attendance was very effective in improving ANC and PMTCT uptake among pregnant women. ${ }^{26}$

\section{Abbreviations And Acronyms}




\begin{tabular}{|ll|}
\hline AIDS & Acquired Immunodeficiency Syndrome \\
\hline ANC & Antenatal care \\
\hline ART & Anti-retroviral therapy \\
\hline AZT & Zidovudine \\
\hline CI & Confidence Interval \\
\hline eMTCT & Elimination of Mother-to-Child Transmission \\
\hline HIV & Human Immunodeficiency virus \\
\hline LIC & Low-income countries \\
\hline MoHCC & Ministry of Health and Child Care of Zimbabwe \\
\hline MRCZ & Medical Research Council of Zimbabwe \\
\hline MTCT & Mother-to-child transmission \\
\hline NAC & National AIDS Council \\
\hline OR & Odds Ratio \\
\hline PMTCT & Prevention of mother-to-child transmission of HIV \\
\hline PrEP & Pre-exposure prophylaxis \\
\hline SdNVP & Zingle dose nevirapine \\
\hline STATA & A statistical analysis software \\
\hline STI & Sexually transmitted infection \\
\hline UNAIDS & Joint United Nations Programme on HIV and AIDS \\
\hline UNICEF & United Nations International Children Emergency Fund \\
\hline WHO & University of Western Cape \\
\hline UWC & ZiMSTAT \\
\hline
\end{tabular}

\section{Declarations}

I declare that this paper entitled: "non-booking for antenatal care and risks for vertical HIV transmission among women in Chitungwiza, Zimbabwe "is an effort by the co-authors and me. It has not been published in any other journal and that all the references I have used or quoted have been acknowledged.

Full name: Patricia Fadzayi Mandima

Date: 03 Dec 2021

Signed:

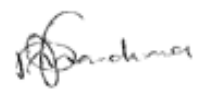

DEFINITION OF KEY TERMS USED IN THIS STUDY 


\begin{tabular}{|ll|}
\hline $\begin{array}{l}\text { Booked } \\
\text { women }\end{array}$ & Refers to women who attended at least one antenatal care visit during a pregnancy. \\
\hline $\begin{array}{l}\text { Unbooked } \\
\text { women }\end{array}$ & $\begin{array}{l}\text { Refers to women who did not have contact with antenatal care services during a pregnancy and only } \\
\text { present at a clinic during labour or after delivery. }\end{array}$ \\
\hline
\end{tabular}

\section{Ethics approval and consent to participate}

Approval for conducting this study was provided by the Bio-Medical Research Ethics Committee at the University of the Western Cape [Ethics reference number: BM/18/1/13], and the Medical Research Council of Zimbabwe [Approval number: MRCZ/B/1549]. Permission to conduct the study in Chitungwiza was granted by the Chitungwiza Ethics Committee.

All methods were carried out in accordance with relevant guidelines and regulations.

The study was a retrospective desk review of de-identified data from clinic records. There was no face-to-face encounter with study participants. Informed consent was therefore waivered by the Bio-Medical Research Ethics Committee at the University of the Western Cape [Ethics reference number: BM/18/1/13], and the Medical Research Council of Zimbabwe [Approval number: MRCZ/B/1549].

\section{Consent for publication}

Consent for publication was given by the Bio-Medical Research Ethics Committee at the University of the Western Cape [Ethics reference number: BM/18/1/13], and the Medical Research Council of Zimbabwe [Approval number: MRCZ/B/1549].

\section{Availability of data and material}

The data can be available upon request and approval by the corresponding author.

\section{Competing interests}

The authors declare that there is no conflict of interest.

\section{Funding}

The study was funded by the author

\section{Authors' Contributions}

Patricia Mandima, Martina Lembani and Nikki Schaay were involved in developing the study proposal and in writing and review of the manuscript. Bernard Ngara was involved in statistical analysis, interpretation of results and manuscript writings. All the authors have read and approved the manuscript.

\section{Acknowledgements.}

We thank the University of Western Cape school of Public Health though whose tutorship I managed to carry out this research. We are also grateful for the technical support received from the University of Zimbabwe- Clinical Trials Research Centre. We acknowledge Livona Chitibura for participating in data collection and Annah Manjengwa for assistance in data entry. Finally we thank nursing staff at the 4 maternity clinics for helping access maternity records.

\section{References}

1. HIV Statistics - Global and Regional Trends - UNICEF DATA. Accessed November 9, 2021. https://data.unicef.org/topic/hivaids/global-regional-trends/ 
2. WHO, 2019. Global Health observatory data: Prevention of mother-to-child transmission (PMTCT). [Online] Available at: https://www.who.int/gho/hiv/epidemic_response/PMTCT_text/en/[Accessed 07 November 2019]

3. WHO, 2010. РMTCT Strategic Vision 2010-2015:. [Online] Available at:

https://www.who.int/hiv/pub/mtct/strategic_vision.pdf[Accessed 10 October 2017)

4. WHO, 2019. Mother-to-child transmission of HIV. [Online] Available at: https://www.who.int/hiv/topics/mtct/en[Accessed 07 November 2019]

5. Lincetto O, Mothebesoane-Anoh S, Gomez P, Munjanja S. (2001). Antenatal care. [Online] Available at: https://www.who.int/pmnch/media/publications/aonsectionlll_2.pdf47

6. WHO, 2016. WHO recommendations on antenatal care for a positive pregnancy experience. [Online] Available at: www.who.int/reproductivehealth/publications/maternal_perinatal_health/ancpositive-pregnancy-experience/en/[Accessed 4 September 2018]

7. UNICEF, 2018. Antenatal care: Only half of women worldwide receive the recommended amount of care during pregnancy. [Online] Available at: https://data.unicef.org/topic/maternal-health/antenatal-care/[Accessed 11 November 2019]

8. ZIMSTAT and ICF International, 2016. Zimbabwe Demographic and Health Survey 2015: Final Report., Rockville, Maryland, USA: ZIMSTAT and ICF International. Available at: www.zimstat.co.zW > sites > files > img > publications > Health > ZDHS_2015

9. Adedokun, S.T., Yaya, S. Correlates of antenatal care utilization among women of reproductive age in sub-Saharan Africa: evidence from multinomial analysis of demographic and health surveys (2010-2018) from 31 countries. Arch Public Health 78, 134 (2020). https://doi.org/10.1186/s13690-020-00516-w

10. AVERT, 2019. Prevention of mother-to-child Transmission of HIV.[Online] Available at:

https://www.avert.org/professionals/hiv-programming/prevention/prevention mother-child\#A comprehensive approach to РMTCT [Accessed 04 Nov 2019].

11. MoHCC, 2017. Elimination of Mother-to-child transmission of HIV and Syphilis 2018-2022. [Online] Available at: http://nac.org.zw/wp-content/uploads/2019/02/Zimbabwe-Elimination-of-Mother-to-Child-Transmission-of-HIV-andSyphillis-2018-2022-1.pdf [ Accessed 05 November 2019]

12. Pell C., 2003. Factors Affecting Antenatal Care Attendance: Results from Qualitative Studies in Ghana, Kenya and Malawi. PLOS ONE, 15 January.

13. Simkhada B., 2008. Factors affecting the utilization of antenatal care in developing countries: systematic review of the literature. JAN, 61(3), pp. 244-260.

14. Ejeta, E. D. (2017, July 7). Factors determining late antenatal care booking and the content of care among pregnant mother attending antenatal care services in East Wollega administrative zone, West Ethiopia. Pan African Medical Journal, 27(184). doi:10.11604/pamj.2017.27.184.10926. eCollection 2017.Bobo F, et al,(2017). Inequities in utilization of reproductive and maternal health services in Ethiopia. Int J Equity Health, 19 June.16(105).

15. Bobo, F, et al,(2017). Inequities in utilization of reproductive and maternal health services in Ethiopia. Int J Equity Health, 19 June.16(105).

16. Tarekegn S. (2014). Determinants of maternal health service utilization in Ethiopia: analysis of the 2011 Ethiopian Demographic and Health Survey. BMC Pregnancy Childbirth, 7 May.14(161).

17. Sinyange NS, et al. (2016). Factors associated with late antenatal care booking: population based observations from the 2007 Zambia demographic and health survey. Pan Afr Med, 24 October.25(109).49

18. Oladokun, A. O.-B. (2010). Proximate predictors of early antenatal registration among Nigerian pregnant women. Pan African Medical Journal, 9(4), 222-5.

19. Galvan, J. W. (2001). Prenatal care utilization and foetal outcomes at Harare Maternity Hospital, Zimbabwe. Central African Journal of Medicine, 47(4), 87-92

20. Chadwick EG, Tierney C, Coletti A, Cotton MF, Ruel TD, Reding CA, Zimmer B, Qin M, Jean-Philippe P, Hazra R, Jackson C, Spector S, Capparelli EV, Mirochnick M, Purdue LT, Costello D, Jennings C, Luzuriaga K, Perlowski C, Bwakura- 
Dangarembizi M, Naidoo KL. (2017). Asymptomatic Hematologic Toxicity with Very Early Combination Antiretroviral Therapy (cART) in In Utero HIV-infected Infants. 9th HIV Paediatrics Workshop. Paris

21. Creswell, J. W.( 2014). The selection of a research approach. In: Research Design. USA: Sage Publications, p. 32.

22. MoHCC and National AIDs Council, 2018. GLOBAL AIDS RESPONSE 2018.

[Online]Availableathttps://www.unaids.org/sites/default/files/country/documents/ZWE_2018_countryreport.pdf[Accessed 07 October 2019].

23. Mahomed K, Kasule J, Makuyana D, Moyo S, Mbidzo M, Tswana S. Seroprevalence of HIV infection amongst antenatal women in greater Harare, Zimbabwe. Cent Afr J Med. 1991 Oct;37(10):322-5. PMID: 1813126.

24. World Health Organisation (WHO). (2016). NCBI. Retrieved October 02, 2017, from https://www-ncbi-nIm-nihgov.ezproxy.uwc.ac.za/books/NBK409099/

25. Stringer, JSA, Sinkala,M, Chapman,V, Edward P. Acosta,a Grace M. Aldrovandi, Mudenda,V, Acosta,EP, Aldrovandi,GM, Stout,JP, Goldenberg,RL, Kumwenda,R, Vermund,SH. (2003). Timing of the maternal drug dose and risk of perinatal HIV transmission in the setting of intrapartum and neonatal single-dose nevirapine. AIDS, 17(11), pp. 1659-1665.

26. Lema IA, Sando D, Magesa L, Machumi L, Mungure E, Mwanyika Sando M, Geldsetzer,P, Foster D, et al. (2014). Community Health Workers to Improve Antenatal Care and PMTCT uptake in Dar es Salaam Tanzania a Quantitative performance evaluation. J Acquir Immune Defic Syndr, 67(4), pp. 195-201. 